\title{
Complexity Theory and Language Development: In celebration of Diane Larsen-Freeman
} Lourdes Ortega \& ZhaoHong Han (Eds.). Amsterdam and Philadelphia: John Benjamins Publishing Company. 2017. Pp. $1+234$.

This book presents a collective effort of scholars in applied linguistics to expand the horizon of the application of Complexity Theory (CT) in the research of second language development (SLD). Ever since the inception of this paradigmatic shift initiated by Diane Larsen-Freeman in 1997, SLD research has gradually transformed to acknowledge, value, and focus on the investigation of the variability and nonlinearity of the SLD process. The curation of this volume includes first a centerpiece by Larsen-Freeman that lays out the theoretical background of CT in SLD research and nine other chapters, both theoretical and empirical, that center around the discussion of SLD as a complex dynamic system. Topics covered include the use of terminology under the CT framework, complex brain structures and language development, research on individual differences, methodological techniques and norms under CT, CT and L2 pedagogy, the multilingual systems, and the ultimate outcome of SLD.

As the theoretical overview and the centerpiece of this volume, Chapter 1 by LarsenFreeman addresses three aspects that are fundamental for understanding language development through the lens of CT, namely what CT is, the paradigmatic shift CT propels in the field of SLD, and three crucial questions if research in SLD were to be guided by such a complex conceptual framework. CT is "fundamentally a theory of change" (p. 12). It provides a conceptual framework, a way of thinking, for us to make sense of the complex phenomena observed in the ever-changing world, be it in the physical or the social realm. Central to CT is the concept of complex systems that are characterized by the five main constructs, namely emergence of patterns, openness, adaptivity, interconnected and context-dependent components, and nonlinearity. As CT has been applied to various domains in scientific research, both in physical and social sciences, Larsen-Freeman puts forward the concept of CT being a transdisciplinary metatheory that has the power of describing and prescribing "the context in which theoretical and methodological concepts are constructed" (Overton, 2007, p. 154) and, therefore, of shifting research paradigms in a given field-SLD in this case. To zoom in on SLD, Larsen-Freeman listed 30 aphorisms concerning language, language learners/users, language learning, and language teaching that reflect a clear conceptual shift away from viewing language as static and language development as involving duality and linear causality. What was celebrated in "the new normal" (p. 28) in SLD was the constant and nonlinear change and the variability exhibited in the developmental trajectory of each individual learner. Larsen-Freeman ends the chapter by addressing three questions most likely asked when the research of SLD is guided by $\mathrm{CT}$. The first question pertains to the challenge posed by discarding the dichotomous thinking so pervasive in the field (e.g., the binary of cognitive versus social approaches to SLD). Larsen-Freeman advocates for allowing paradoxes, in order to appreciate the non-duality of the world and the complementarity of the two seemingly polarized members of a pair. The second and the third questions concern practical issues including the boundary problem (LarsenFreeman, 2011) and the tension between rejecting linear causality and generalizing research

(C) 2019 Zhang. This is an open access article distributed under the terms of the Creative Commons Attribution License, which permits the user to copy, distribute, and transmit the work provided that the original authors and source are credited. 
findings. Although identifying the boundary between a system and its environment indeed seems against the interconnectedness and interaction among all things, which complexity thinking endorses, Larsen-Freeman suggests that drawing the line should be a strategic endeavor on the basis of understanding the complex whole and should depend on the research interest at hand. In terms of generalizability of research findings under CT, Larsen-Freeman aptly asserts that the contingent nature of SLD, manifesting itself as nonlinearity development, "does not preclude generalizing" (p. 34). With complexity thinking, generalizations should be made about the dynamics in the system under study as opposed to about any single interventional factor and its possible linear effect to a much larger population of language learners.

Before embarking upon the discussion of SLD under CT, the next chapter by de Bot first clarifies the interchangeable use of the two terms of CT and Dynamic Systems Theory (DST) commonly observed so far in applied linguistics (AL). While both CT and DST attempt to account for the behavior of dynamic complex systems, CT primarily pertains to epistemology involving the ontology of issues such as self-organization and emergentism. Or, to echo back to the first chapter, CT is a metatheory. On the other hand, DST has a rather mathematical origin and is originally concerned more about hard sciences. De Bot then points out that there are two basic approaches to describing dynamic systems - metaphorical (i.e., dynamic systems) and mathematical (i.e., dynamical systems) — and the latter provides a mathematical vocabulary for characterizing the behavior of dynamic systems, e.g., fractals, bifurcation, and attractors. In the context of AL, both theories consider human cognition and language learning dynamic, adaptive, and complex, yet DST offers several analytical tools for AL researchers to deal with dense longitudinal language data for studying the development over time, such as min/max analysis, Monte-Carlos analyses, and time series analysis. Therefore, a new term of Complex Dynamic Systems Theory (CDST) is proposed to encompass both the metaphorical and technical approaches and this new term in fact conveys an intention to apply the metaphorical complexity thinking through the implementation of technical research tools.

In Chapter 3, Schumann discusses the complexity of neural structures and functions and of language use. In an analogous sense, both the brain and the human language are degenerate in that different neural structures that could produce the same function and different linguistic elements can also produce the same message. Degeneracy, according to Schumann, goes "handin-hand" with complexity (p. 61), because components of a complex system become integrated as a result of their interconnectedness and such integration allows different systems to produce the same or similar output. Here Schumann illustrates such degeneracy in complexity systems by drawing on the motivation research in second language acquisition (SLA). On the one hand, instead of a one-to-one mapping between the five stimulus appraisals which all motivational constructs boil down to and the regions in the neural system where these appraisals are implemented, what has emerged in motivational research is degeneracy, that is, a many-to-many mapping between subcomponents of the neural system and the appraisals' underlying motivational constructs. As a result, no functions could be pinpointed to a single region of the neural system and the function of one brain region could not be simply predicted based on the activity observed for one task. On the other hand, the degenerate lexicon of human language aggravates the issue in that the concept of motivation tends to be labeled with synonyms as it is researched in different relevant areas - educationists study motivation in the sense of "interest," "will," or "motives," whereas psychologists label it as "wants," "rewards," or "energy." In a 
nutshell, the physical structure of the brain and its functions are two complex dynamic systems and the attempt of labeling their complex mappings using the human lexicon, which itself is also complex and dynamic, is bound to be unsuccessful.

Indeed, the research on motivation and its superordinate topic-learner characteristicsin the field of SLA has been one of the most fruitful sub-areas implementing CDST. In Chapter 4, Dörnyei first urges us to challenge the traditional view on individual differences (IDs) - $\mathrm{a}$ view that considers these mediating factors (i.e., motivation, aptitude, learning style, learning strategies, etc.) in language development as stable and distinct traits, that believes the parameters of these factors can be measured quantitatively and used to make predictions about language development, and that holds that there is a linear causality between these factors and the learner's language development. This line of conceptualizing IDs, despite its pervasiveness in our field, is not compatible with the holistic and dynamic CDST perspective and a new framework is therefore called for. By adapting McAdams and Pals' (2006) Big Five model, Dörnyei proposes a new framework involving three levels of personality, namely dispositional traits, characteristic adaptations, and integrative life narratives. Dispositional traits are the relatively stable and decontextualized dimensions of personality largely genetically determined, e.g., friendliness or extraversion, whereas characteristic adaptations, such as motives, values, self-images, and goals, are constructs highly contextualized by the environmental conditions. Integrative life narratives refer to a "highly personal organisational framework" (p. 86) that a person uses to make sense of their own life, forming what McAdams and Pals (2006) call the narrative identity. In the context of SLA, Dörnyei explains that although a learner's dispositional traits are mostly stable, their impact on SLD is usually mediated through characteristic adaptations. Furthermore, research has shown that integrative life narratives could play even a more powerful role in altering a learner's perception of their learning experience, i.e., they narrate themselves into the self they become. This new framework views IDs as complexes involving multiple components, respects the dynamic interplay among these components within the same level and also across levels, and acknowledges the situational variation IDs display.

Chapter 5 continues the discussion on studying IDs as complex dynamic systems by tackling the methodological challenges. McIntyre, MacKay, Ross, and Abel argue that the shift towards complexity thinking in the field of SLD has led to a metaphorical conceptual framework to generate new questions, whereas a rigorous empirical research program to help researchers deal with the dense, longitudinal, and individual data for answering these questions has yet to be well developed. To address this issue, McIntyre et al. review 12 methods that have been applied to empirical studies on motivation. These methods, from qualitative, mixed, to quantitative, are: (1) longitudinal qualitative interview design, (2) retrodictive qualitative modeling, (3) qualitative comparative analysis, (4) two-stage qualitative interview design, (5) qualitative interview design gathering data on multiple timescales, (6) longitudinal cluster analysis, (7) mixed-methods research/triangulation of multiple data sources, (8) Q methodology, (9) trajectory equifinality model, (10) idiodynamics, (11) latent growth modelling, and (12) change point analysis.

Following the brief review of these 12 research methods is an apt discussion in Chapter 6 by Lowie regarding the quality norms for empirical studies adopting a dynamic approach. Lowie points out that what is yet to be built when applying a CDST approach in empirical SLD research is a set of conventions and requirements for this type of research in order to accurately unravel 
the variability and nonlinear change in SLD. Lowie suggests conducting process-oriented research, as opposed to product-oriented research where the time dimension of development cannot be addressed, that draws evidence from observations of individuals over time to analyze person-specific processes. Two research methods are proposed: the microgenetic method for empirically analyzing intra-learner variability and the dynamic modelling by computer simulations for investigating complex dynamic relationships between subsystems. Lowie asserts that these two methods used in combination have great potential so long as researchers provide a rationale for the density of data collection and the time scale of the measurements. To address concerns over the lack of generalizability, Lowie contends that the fact that results from longitudinal case studies cannot be generalized to a larger population does not reduce the quality and the value of research; and replication, instead of generalization, is more relevant to case studies in that the patterns and relationships found in multiple case studies would better advance the understanding of SLD as a dynamic system.

This perspective on SLD as a complex dynamic system not only has pushed the field to explore new paths for researching and theorizing, it also has crucial implications for language pedagogy in that, as Verspoor argues in Chapter 7, it is highly informative about what to teach and how to teach. By drawing on the dynamic usage-based (DUB) approach, Verspoor proposes the term Form-Use-Meaning-Mappings (FUMMs) to refer to the units of language, as this new term highlights the complex nature of language that involves dynamically interrelated subsystems (i.e., phonology, morphology, syntax, semantics, and pragmatics). Verspoor therefore contends that FUMMs should be the units of teaching because communication - the ultimate goal for learning a language - emerges through "the use of FUMMs in meaningful contexts in which humans have to dynamically co-construct meaning" (p.150). As for how they ought to be taught, Verspoor aptly warns us that learners themselves are also complex systems and intensive exposure to the target FUMMs in meaningful contexts through iteration is desirable in order for them to reach self-organization. Here she explains that iteration refers to the combination of repetition and recursion that affords the variability through which the learners can selectively reorganize the patterns that emerge into their own systems.

The next two chapters review previous empirical research adopting other approaches through the CDST lens. Chapter 8 by Opitz revisits four previous empirical studies of multilingual development that investigate the learning, relearning, and maintaining of a second language (L2) and also the development of the participants' first languages (L1s). All four studies differ in their original purpose and basic design, but they share an underlying conceptualization that each participant's multilingual repertoire is a dynamic system consisting of both L1 and L2 skills that interact with each other and with the context in which they are used. Through analyses of both linguistic and perception data on different timescales of weeks, months, and years, the four studies converge at several important findings. First, the multilingual system as a whole displays constant variability in all languages involved on all timescales although at times some language(s) may exhibit developmental stasis. Second, the developmental patterns of the same participant across timescales are non-linear. For example, in the last study on foreign language learners of Russian, it was observed that the same learner's error rate on the same construction first fluctuated from month to month, then reached peak accuracy, but experienced clear backsliding after only a one-week break. The third finding highlights that language use and context play a strong role in the development of a multilingual 
system. Specifically, an authentic language use context seems to have always exerted positive effects on the language called for, be it improving an L2 or maintaining an L1; on the other hand, when the context for language use is absent, L2 loss or L1 attrition could happen.

These findings are corroborated in Chapter 9 by Köpke who discusses three situations of language destabilization drawing on findings from neuroscience and neuropsycholinguistic research: (1) when changes in language use patterns lead to attrition, (2) when adding a new language results in the reorganization of a multilingual system, and (3) when aphasic patients adapt to acquired language disorders. Köpke argues that the lack of L1 use could induce changes internal to the linguistic system and the changes to L1 use, or, say, the replacement by L2 in certain contexts could lead to changes in the corresponding functional domains. It is precisely the interaction of these internal and external processes, involving multiple factors such as age, length of residency, frequency of L1 use, and type of L1 use, that gives rise to the emerging L1 attrition. As for adding a new language to a user's language system, evidence from both the brain's electrical activity measured by ERPs and gray-matter density measured by MRI has supported that: The brain structure experiences the greatest changes at the early stages of learning a new language, the existing language system will be temporarily destabilized, and the brain structure will eventually adapt to the new system. Finally, a holistic systems approach has been applied to aphasia (i.e., acquired language disorders), taking into account both the patients' adaptive verbal behavior and the variability observed in their deficit. While the adaptive behavior proves, again, that the brain is a highly adaptive dynamic system, the variability that mono- and bilingual patients show in their behavior sheds light on the insight that language functions may very well be intertwined with other cognitive functions (e.g., the executive function).

Following an extensive discussion on the various local aspects of SLD is the last chapter by Han, Bao, and Wiita that zooms out to address a global issue of the inter-learner differential ultimate attainment. The authors argue that seeking for simplicity in the complex system of SLD is not only possible but also necessary as a complexity perspective allows for nuanced observation and description; but a simplicity perspective affords explanation and prediction. The reasoning follows that while SLD is indeed open, adaptive, and self-organizing if viewed through the CDST lens at a microscopic developmental level, as a whole SLD is rather closed and predictable of its outcome when zooming out to a macroscopic level. For this reason, the complexity and simplicity perspectives should be seen as complementary as opposed to mutually exclusive. To address the closeness of SLD, the authors seek wisdom from the classical physics law of Energy Conservation Theory (ECT) and present the Energy Conservation Theory in L2 (ECT-L2), a new theoretical framework that maps the four key variables of L2 learningmotivation, aptitude, L1-Target Language (TL) distance, and TL input - onto the formula of ECT, both metaphorically and mathematically. ECT holds that "energy can be converted from one form to another, but the sum of all forms of energy in a closed system remains constant" ( $p$. 216). Metaphorically, motivation and aptitude are the operationalization of kinetic energy, L1TL distance that of the centrifugal energy, and traction of TL input that of the potential energy. Mathematically, the ECT formula would predict the transformation of energies as the object travels through the field towards the target, i.e., how the variables interact as the learner progresses in learning the L2. According to ECT-L2, motivation and aptitude initially drive the learning to commence; as the learner's proficiency level increases, the traction of TL input exerts greater influence, but the L1-TL distance eventually holds the learner back from approaching the 
target. All four variables clearly play a dynamic role in the developmental process, and the dynamic interaction between them, which varies from learner to learner, leads to inter-learner differential ultimate attainment.

This volume addresses the theoretical, methodological, and empirical facets of SLD research under the new conceptual framework of CT (or CDST for that matter). On the one hand, it offers a thoughtful and comprehensive update on the newest paradigmatic shift towards complexity and systems thinking, which can be highly informative to researchers new to CT. On the other hand, the methodological discussion in this book provides researchers already adopting a CDST approach with useful resources for conducting future empirical studies. Although the contributors do not completely agree on all accounts of CT mentioned in this book, what has emerged is the complementarity of their views. This theoretical pluralism enabled by an overarching metatheory of CT would promote the healthy development of SLD research in the long run.

\section{RAINIE (MINGHAO) ZHANG}

Teachers College, Columbia University

\section{REFERENCES}

Larsen-Freeman, D. (1997). Chaos/complexity science and second language acquisition. Applied Linguistics, 18, 141-165.

Larsen-Freeman, D. (2011). A complexity theory approach to second language acquisition/development. In D. Atkinson (Ed.), Alternative approaches to second language acquisition (pp. 48-72). Oxford, UK: Oxford University Press.

McAdams, D. P., \& Pals, J. L. (2006). A new Big Five: Fundamental principles for an integrative science of personality. American Psychologist, 61, 204-217.

Overton, W. F. (2007). A coherent metatheory for dynamic systems: Relational organicism-contextualism. Human Development, 50, 154-159. 